\title{
Effect of selected biofilm inhibitors, $N$-acetylcysteine and DNase, on some biological properties of taurine haloamines (TauCl and TauBr)
}

\author{
MARIA WALCZEWSKA ${ }^{1}$, ANNA BIAEECKA ${ }^{2}$, ANNA GACON $^{3}$, EWA PASICH ${ }^{3}$, \\ ANDRZEJ KASPROWICZ2, JANUSZ MARCINKIEWICZ ${ }^{3}$ \\ ${ }^{1}$ Jagiellonian University Medical College, Krakow, Poland \\ ${ }^{2}$ Centre of Microbiological Research and Autovaccines Ltd., Krakow, Poland \\ ${ }^{3}$ Department of Immunology Jagiellonian University Medical College, Krakow, Poland
}

\begin{abstract}
Antibiotic resistance is a common problem accompanying biofilm-associated chronic infections. New therapeutic strategies are based on a combined application of antiseptics with anti-biofilm agents. Taurine haloamines, taurine chloramine ( $\mathrm{TauCl}$ ) and taurine bromamine (TauBr), show antimicrobial and anti-inflammatory properties, which have been examined in a variety of local infections, including biofilm-associated infections. In contrast to beneficial antimicrobial effects of taurine haloamines against the planktonic form of bacteria, their efficacy against bacteria hidden in biofilm need to be enhanced. One possibility is to use them together with agents capable of destroying components of biofilm matrix. In this study we ask a question whether TauCl or TauBr are effective in killing Streptococcus mutans and Porphyromonas gingivalis, major oral bacteria responsible for the development of dental plaque and pathogenesis of periodontal diseases. Moreover, we have examined TauBr and TauCl stability in the presence of $N$-acetylcysteine (NAC) and DNase, agents with known anti-biofilm activity. We have found that TauBr was much stronger than TauCl microbicidal agent against both tested bacterial strains. However, TauBr was less stable than TauCl. NAC readily decomposed TauBr but not TauCl. In addition, TauBr inhibited DNase activity, when used in excess. This preliminary study confirms previous opinions that taurine haloamines have great potential in killing oral bacteria. However, further studies are necessary to find anti-biofilm agent( $s)$ which together with TauCl/TauBr will give at least an additive therapeutic effect in the treatment of chronic infections, to support or replace ineffective antibiotic therapy.
\end{abstract}

Key words: biofilm, $N$-acetylcysteine (NAC), DNase, taurine bromamine (TauBr), taurine chloramine (TauCl), oral bacteria, periodontal diseases.

(Centr Eur J Immunol 2013; 38 (4): 434-442)

\section{Introduction}

Taurine chloramine $(\mathrm{TauCl})$ and taurine bromamine (TauBr), the major haloamines of the MPO-halide system of neutrophils, exert anti-microbial and anti-inflammatory properties, as documented in a number of in vitro studies [1-11]. Importantly, clinical studies have shown that both haloamines are also effective in the local treatment of inflammatory diseases, including biofilm-related infections, such as otitis externa, chronic rhinosinusitis [13, 14] or acne vulgaris [15]. Moreover, $\mathrm{TauCl}$ has been proposed as a novel therapeutic agent in the treatment and prevention of periodontal diseases, chronic infectious diseases related to the excessive development of the polymicrobial oral biofilm [16]. However, mature biofilms, consortia of bacteria attached to biological surfaces (e.g. dental plaque) and enclosed in self-generated matrix, are characterized by a high resistance to antibiotics and antiseptics. Therefore, new alternative therapeutic strategies should be tested. One possibility is to use antiseptics along with anti-biofilm agents to increase effectiveness of antimicrobial therapy $[17,18]$.

Only recently we have reported that $\mathrm{TauBr}$ is able to inhibit in vitro the formation of the Pseudomonas aeruginosa biofilm, but cannot eradicate the mature biofilm and 
cannot effectively kill bacteria hidden in the biofilm matrix [18]. Therefore, it is tempting to test the anti-biofilm effect of taurine haloamines in combination with agents which can destroy components of biofilm matrix (extracellular polysaccharides and/or DNA).

The aim of this study was to investigate the effect of $\mathrm{N}$-acetylcysteine (NAC) and DNase, well-documented anti-biofilm agents, on stability and some biological properties of taurine haloamines. This laboratory study design is the first step in testing therapeutic potential of taurine haloamines combined with other anti-biofilm agents for a local treatment of biofilm-associated chronic infections, such as periodontal diseases.

\section{Material and methods}

\section{Anti-biofilm agents}

$\mathrm{N}$-acetylcysteine [2-Acetamido-3-sulfanylpropanoic acid] (NAC), bovine pancreatic DNase I (DNase). Both reagents purchased from Sigma, Aldrich, Germany.

\section{Taurine haloamines: taurine chloramine (TauCl) and taurine bromamine (TauBr)}

$\mathrm{TauCl}(N$-chlorotaurine sodium salt) a kind gift from Professor Waldemar Gottardi and Professor Marcus Nagl from the Division of Hygiene and Medical Microbiology, Innsbruck Medical University, Austria. TauCl as a crystalline sodium salt (molecular weight 181.57) was prepared as described previously [1]. Each preparation of TauCl was monitored by UV absorption spectra ( $\lambda=200$ to $400 \mathrm{~nm})$ to assure the authenticity of $\mathrm{TauCl}(\lambda=252 \mathrm{~nm})$ and the absence of dichloramine $\left(\mathrm{TauCl}_{2}\right)(\lambda=300 \mathrm{~nm})$ and unreacted $\mathrm{HOCl} / \mathrm{OCl}^{-}(\lambda=292 \mathrm{~nm})$. The concentration of synthesized TauCl was determined using the molar extinction coefficient $429 \mathrm{M}^{-1} \mathrm{~cm}^{-1}$ at $\mathrm{A}_{252}$.

$\mathrm{TauBr}$ was prepared in a two-step procedure. First, $\mathrm{NaOBr}$ was synthesized in reaction between equimolar amounts of $\mathrm{NaOCl}$ and $\mathrm{NaBr}(P O C H$, Poland) in the PBS solution. In such conditions virtually all the $\mathrm{OCl}^{-}$present reacts with $\mathrm{Br}^{-}$to form $\mathrm{OBr}^{-}$and $\mathrm{Cl}^{-}$. The presence and concentration of $\mathrm{OBr}^{-}$was confirmed by $\mathrm{UV}$ spectra $(\lambda=$ $=200$ to $400 \mathrm{~nm}$ ). In the second step, $20 \mathrm{mM} \mathrm{NaOBr}$ was added dropwise to an equal volume of $400 \mathrm{mM}$ taurine. UV absorption spectrum was checked to exclude the formation of taurine dibromamine or chloramines and to estimate the concentration of $\mathrm{TauBr}$ (molar extinction coefficient -430 $\mathrm{M}^{-1} \mathrm{~cm}^{-1}$ at $\mathrm{A}_{288}$ ). Stock solutions of $\mathrm{TauCl}$ and $\mathrm{TauBr}$ were kept at $4^{\circ} \mathrm{C}$ for a maximum period of 3 days before use.

\section{Bacterial strains}

All tests were performed on Streptococcus mutans ATCC 35668 (S. mutans) and Porphyromonas gingivalis ATCC 33277 (P. gingivalis).

\section{Animals}

The study was performed on CBA male/female mice, between 6 and 8 weeks of age, from the breeding unit, Department of Immunology UJ CM, Kraków. The mice were fed commercial, granulated food and water ad libitum. The study protocol was approved by the Local Ethics Committee in Kraków (No. 91/2011).

\section{Peritoneal exudate cells}

Peritoneal exudate cells (PECs) isolated from CBA mice were induced by intraperitoneal injection of $1.0 \mathrm{ml}$ of thioglycolate. The cells were collected $18 \mathrm{~h}$ later by washing out the peritoneal cavity with $5 \mathrm{ml}$ of PBS (phosphate buffer solution) containing $5 \mathrm{U}$ heparin $/ \mathrm{ml}$. Then the cells were centrifuged and red blood cells were lysed by osmotic shock using distilled water; osmolarity was restored by addition of $2 \times$ concentrated PBS.

\section{Measurement of TauCl and TauBr stability}

To determine the influence of anti-biofilm agents on stability of taurine haloamines, $\mathrm{TauBr}$ and $\mathrm{TauCl}$ were mixed with equimolar concentrations of NAC or DNase ( $3 \mathrm{mM}$ ). Reagents were kept in PBS for 60 minutes and then the stability of the tested agents was evaluated by analysis of their UV spectra, as described above.

\section{Analysis of DNAse activity}

\section{Digestion of plasmid DNA with DNase I in the presence of TauCl or TauBr}

One microgram of plasmid DNA pEGFP (Clontech) (6500 base pairs) was digested in 20 microliters of the reaction mixture, containing one microgram of DNase I (2.15 Kunitz units) and varied concentrations of $\mathrm{TauCl}$ or Tau$\mathrm{Br}$, in the presence of $40 \mathrm{mM}$ Tris- $\mathrm{HCl}(\mathrm{pH} 8.0), 10 \mathrm{mM}$ $\mathrm{MgSO}_{4}$ and $1 \mathrm{mM} \mathrm{CaCl}_{2}$. Reactions were carried out for 40 minutes at $37^{\circ} \mathrm{C}$ and terminated by a thermal inactivation of DNase I (10 minutes at $\left.70^{\circ} \mathrm{C}\right)$.

\section{Electrophoresis}

Digestion mixtures were separated by electrophoresis in $0.8 \%$ agarose gel, under constant voltage (4V per centimeter of distance between electrodes). Tris-borate buffer was used as a conductive medium $(10.8 \mathrm{~g} / \mathrm{l}$ Tris base, $5.5 \mathrm{~g} / \mathrm{l}$ boric acid, $10 \mathrm{mM}$ EDTA pH 8.0) and ethidium bromide was used for DNA visualization. Images were collected with UV transiluminator (Vilber Lourmat) and BioCapt 97.05s software.

\section{Antimicrobial activity of the tested agents}

Streptococcus mutans strain was grown in a Mueller-Hinton II Agar (Difco, USA) with 5\% sheep blood addition at $35^{\circ} \mathrm{C}$ for 72 hours in aerobic conditions. P. gin- 
givalis was grown in a Schaedler Agar Base (Difco, USA) at $35^{\circ} \mathrm{C}$ for 7 days in anaerobic conditions. Bacteria were centrifuged at $1800 \times \mathrm{g}$, washed twice with $0.9 \% \mathrm{NaCl}$ and diluted in saline to a concentration of $1 \times 10^{8}$ c.f.u./ $\mathrm{ml}$. Before use, bacteria were diluted in a phosphate buffer $(\mathrm{PBS})(\mathrm{pH} 7.4)$ to achieve a final concentration of $1 \times 10^{5}$ c.f.u./ml and then incubated with different concentrations of $\mathrm{TauBr}$ and $\mathrm{TauCl}$. Immediately after the incubation (30 min), aliquots were removed and the viable cell count was determined by a pour-plate method, as described previously [2].

\section{Measurement of reactive oxygen species generation: luminol-dependent chemiluminescence}

The effect of TauCl, TauBr and NAC on the generation of reactive oxygen species (ROS) by neutrophils was evaluated in vitro using luminol-dependent chemiluminescence (LCL). LCL was counted at $37^{\circ} \mathrm{C}$ in temperature-stabilized luminometer Lucy 1 (Anthos, Austria). Briefly, 18hour PECs induced by thioglycolate $\left(5 \times 10^{5} /\right.$ well $)$, were

Table 1. Minimal inhibitory concentration of $\mathrm{TauCl}$ and TauBr [19]

\begin{tabular}{lcc}
\hline $\begin{array}{l}\text { Minimal inhibitory } \\
\text { concentration MIC] }\end{array}$ & S. mutans & P. gingivalis \\
\hline $\mathrm{TauCl}$ & $3 \mathrm{mM}$ & $188 \mu \mathrm{M}$ \\
\hline $\mathrm{TauBr}$ & $24 \mu \mathrm{M}$ & $48 \mu \mathrm{M}$ \\
\hline
\end{tabular}

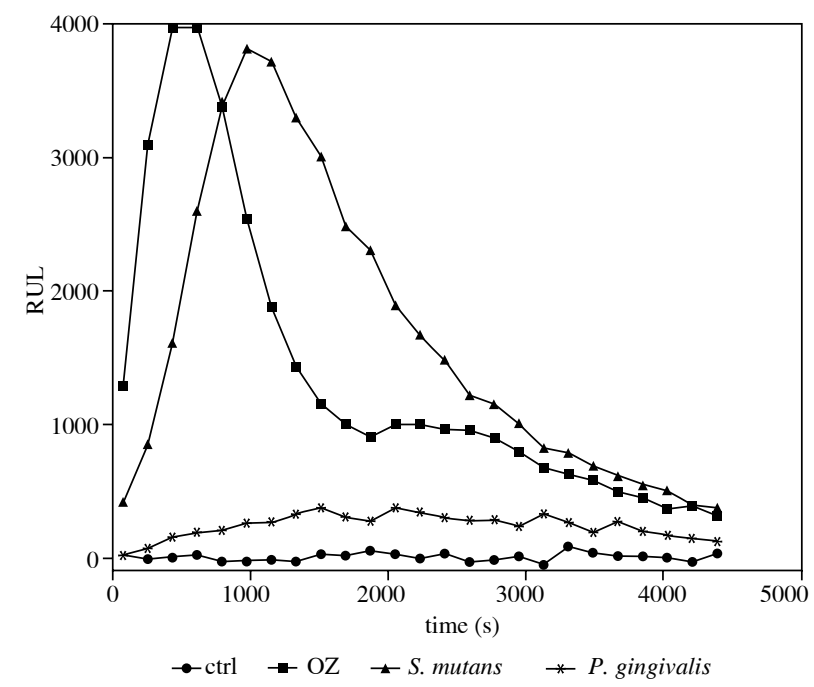

Fig. 1. Generation of ROS by PECs stimulated with opsonized zymosan (OZ) or killed bacteria (S. mutans or $P$. gingivalis). Ctrl - non-stimulated cells. LCL was performed and measured as described in methods. The figure shows one representative experiment preincubated with tested agents in Hank's balanced salt solution $\left(10 \mathrm{~min}\right.$ at $37^{\circ} \mathrm{C}$ in an atmosphere of $\left.5 \% \mathrm{CO}_{2}\right)$ on a 96-well flat-bottom black plate (Nunc, Denmark). Then, the cells were mixed with luminol $(0.8 \mathrm{mg} / \mathrm{ml})$ at the $1: 1$ volume ratio (both Sigma-Aldrich, Germany) and incubated at $37^{\circ} \mathrm{C}$ for another $30 \mathrm{~min}$. After incubation, the cells with TauCl, TauBr and/or NAC were immediately stimulated with opsonized zymosan $200 \mu \mathrm{g} / \mathrm{ml}$ (Sigma-Aldrich, Germany) or killed bacteria (S. mutans or $P$. gingivalis) at a ratio of $100: 1$ (bacteria to the cells). Photon emission over 75 min with 3-min intervals was measured. Results are expressed as relative unit light (RUL) where photons were counted every 5 seconds.

\section{Results}

\section{Antimicrobial capacity of TauCl, TauBr against S. mutans, $P$. gingivalis}

To confirm that taurine haloamines are good candidates for the treatment of periodontal diseases, their antimicrobial activity against the planktonic form of selected dental plaque bacteria was tested in vitro.

$\mathrm{TauCl}$ presents much weaker antimicrobial potential against tested bacterial strains than $\mathrm{TauBr}$, as shown in Table 1. Minimal inhibitory concentration (MIC) of TauCl

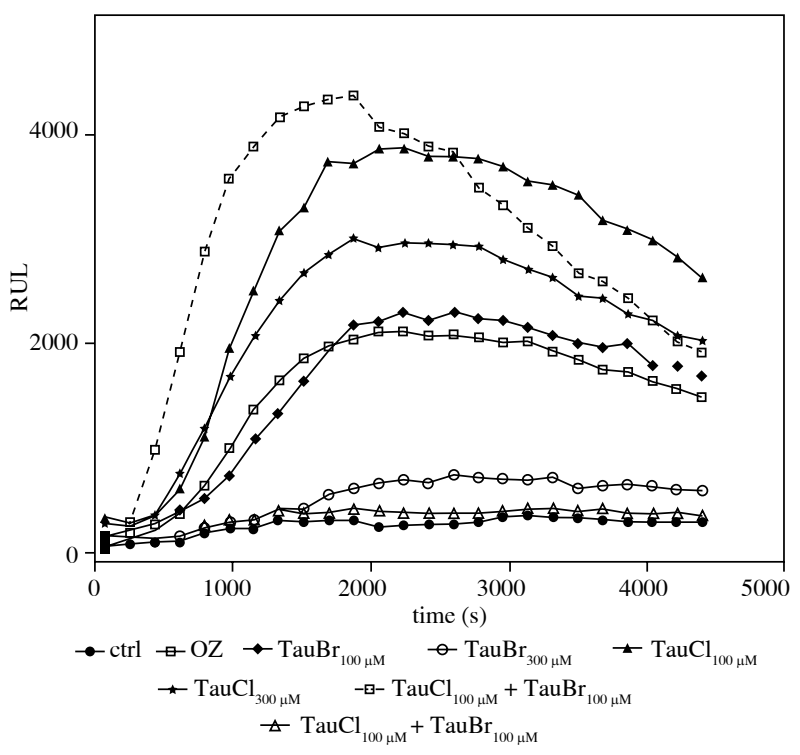

Fig. 2. Effect of $\mathrm{TauCl}$ and $\mathrm{TauBr}$ on ROS production by PECs stimulated with opsonized zymosan. PECs $\left(5 \times 10^{5} /\right.$ well), were preincubated with the tested agents, $\mathrm{TauCl}$, $\mathrm{TauBr}$ or $\mathrm{TauCl}+\mathrm{TauBr}$ for 10 minutes. Then, LCL was performed and measured as described in methods. Ctrl non-stimulated cells. OZ - stimulated cells, positive control LCL $=100 \%$. The figure shows one representative experiment 
for $P$. gingivalis was $\sim 3.5$ times higher than that of Tau$\mathrm{Br}$. Much bigger differences between $\mathrm{TauBr}$ and $\mathrm{TauCl}$ antimicrobial activities against $S$. mutans were observed. MIC of TauBr was $188 \mu \mathrm{M}$ while TauCl effectively killed $S$. mutans at concentrations above $3 \mathrm{mM}$.

\section{Generation of ROS by PECs in the presence of TauCl, TauBr and NAC}

To determine whether tested agents affect ROS generation by inflammatory cells, peritoneal exudate cells were stimulated either with opsonized zymosan (OZ) or bacteria (S. mutans, $P$. gingivalis) in the presence of the agents. Both, opsonized zymosan and $S$. mutans, induced massive generation of ROS, as measured by LCL (Fig. 1). In contrast, $P$. gingivalis, an anaerobic bacterial strain, was a much weaker inducer of ROS generation (Fig. 1).

Both haloamines show a significant, dose-dependent reduction of LCL induced with all stimuli (OZ - Fig. 2; $S$. mutans, $P$. gingivalis - Fig. 4, Table 2), with the strong additive effect when added together (inhibition $>90 \%$ ). At the used concentrations NAC was a much weaker antioxidant than both haloamines. Importantly, there was no additive antioxidant effect when NAC was incubated together with TauCl (Fig. 3 and Fig. 5B). DNase did not affect ROS generation (data not shown).

\section{Effect of NAC and DNase on the stability of TauCl and TauBr}

To answer the question whether NAC and DNase affect stability of $\mathrm{TauCl}$ and $\mathrm{TauBr}$ and in consequence af-

A

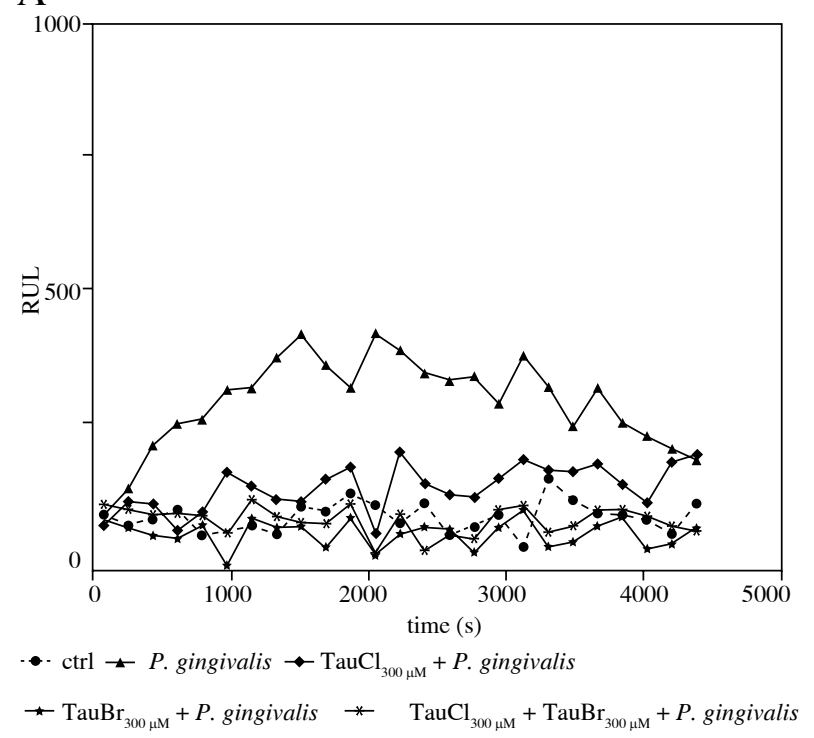

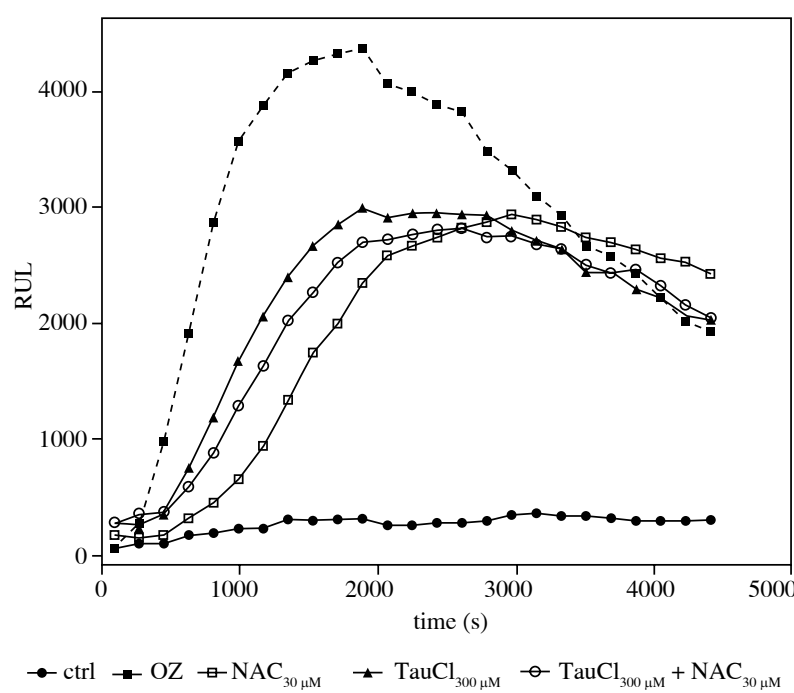

Fig. 3. Effect of TauCl and NAC on ROS production by PECs stimulated with opsonized zymosan. PECs $\left(5 \times 10^{5} \%\right.$ well) were preincubated with tested agents $\mathrm{TauCl}, \mathrm{NAC}$ and $\mathrm{TauCl}+\mathrm{NAC}$ for 10 minutes. Then, LCL was performed and measured as described in methods. Ctrl - control, non-stimulated cells. OZ - stimulated cells. The figure shows one representative experiment

fect their expected biological properties, the UV spectra were analyzed. As shown in Fig. 6, at neutral pH the UV spectrum of TauBr disappeared after NAC addition, while there was no influence of NAC on the UV spectrum of $\mathrm{TauCl}$ when both reagents were used at the equimolar con-

$\mathrm{B}$

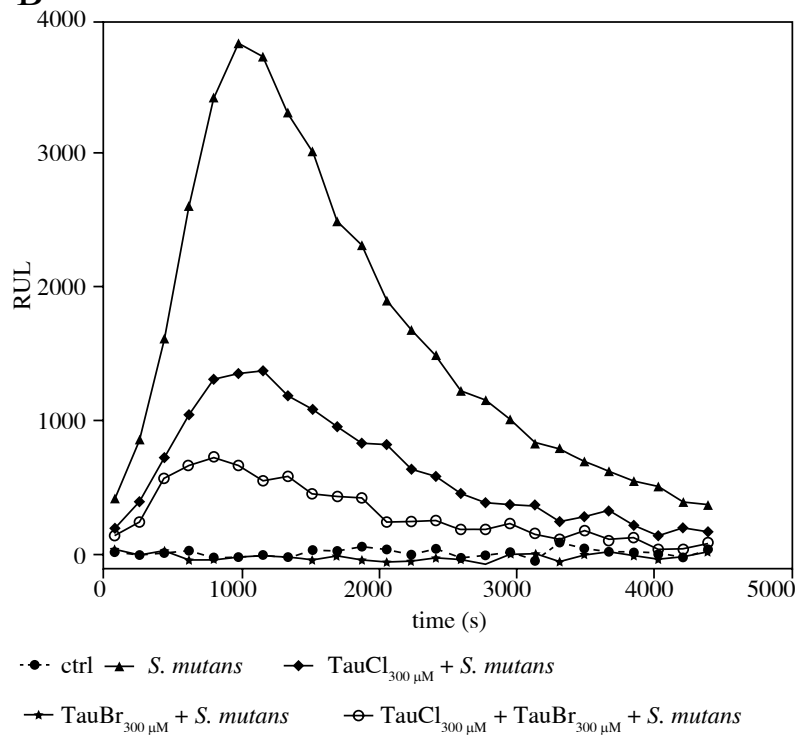

Fig. 4. Effect of TauCl and TauBr on ROS production by PECs stimulated with P. gingivalis (A) or S. mutans (B). PECs $\left(5 \times 10^{5} /\right.$ well $)$ were preincubated with $\mathrm{TauCl}$ and/or $\mathrm{TauBr}$ for 10 minutes. Then, LCL was performed and measured as described in methods. Ctrl - control, non-stimulated cells. The figure shows one representative experiment 
Table 2. The inhibitory effect of TauCl, TauBr and NAC on the ROS production by PECs

\begin{tabular}{|c|c|c|c|c|c|c|c|c|}
\hline \multicolumn{9}{|c|}{$\%$ inhibition of ROS production* } \\
\hline & TauCl $_{100}$ & $\mathrm{TauCl}_{300}$ & $\mathrm{TauBr}_{100}$ & $\mathrm{TauBr}_{300}$ & $\begin{array}{c}\text { TauCl }_{100} \\
+ \\
\text { TauBr }_{100}\end{array}$ & $\begin{array}{c}\mathrm{TauCl}_{300} \\
+ \\
\mathrm{TauBr}_{300}\end{array}$ & $\begin{array}{c}\mathrm{TauCl}_{300} \\
+ \\
\mathrm{NAC}_{30}\end{array}$ & $\mathbf{N A C}_{30}$ \\
\hline $\begin{array}{l}\text { Induction of ROS } \\
\text { generation with opsonized } \\
\text { zymosan }\end{array}$ & 8 & 27 & 47 & 83 & 48 & 90 & 35 & 31 \\
\hline $\begin{array}{l}\text { Induction of ROS } \\
\text { production with } S \text {. mutans }\end{array}$ & - & 72 & - & 64 & - & 98 & 74 & 35 \\
\hline
\end{tabular}

*\% inhibition has been calculated from the results shown in Figs. 1-5

A

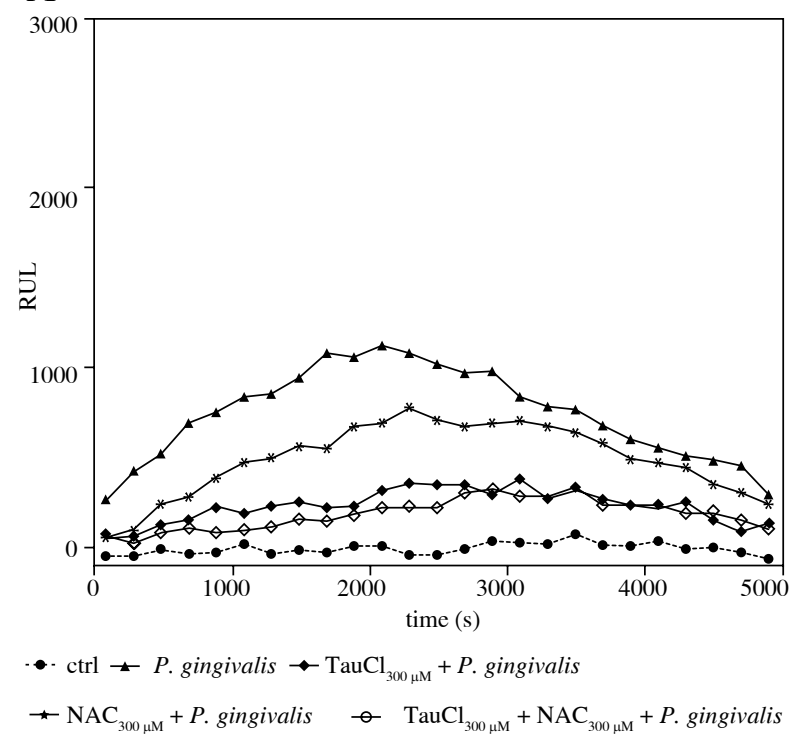

B

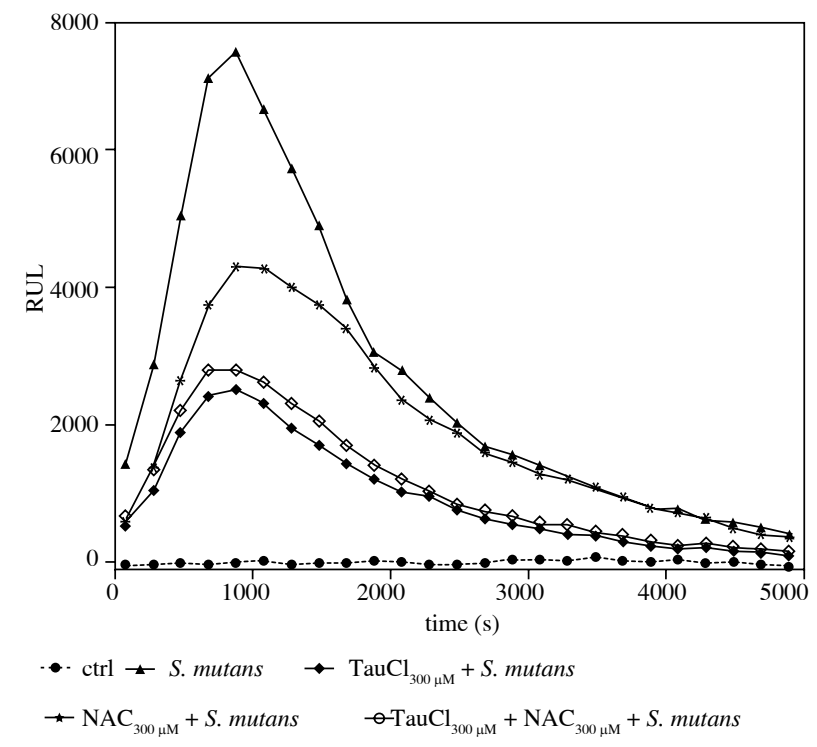

Fig. 5. Effect of TauCl and NAC on ROS production by PEC stimulated with $P$. gingivalis (A) or $S$. mutans (B). PECs $\left(5 \times 10^{5} /\right.$ well $)$ were preincubated with the tested agents TauCl and/or NAC for 10 minutes. Then, LCL was performed and measured as described in methods. Ctrl - control, non- stimulated cells. The figure shows one representative experiment

centrations. DNase had no effect on UV spectra of TauCl and $\mathrm{TauBr}$ (Fig. 7). These results clearly indicate that NAC but not DNase strongly affects stability of TauBr.

\section{Effect of TauCl and TauBr on the activity of DNase}

To examine the effect of TauCl and TauBr on DNase activity, plasmid DNA was digested with DNAse alone or with DNase in the presence of various concentrations of $\mathrm{TauCl}$ or TauBr. Digestion mixtures were separated by electrophoresis in $0.8 \%$ agarose gel as described in methods. DNAse used at a concentration of $50 \mu \mathrm{g} / \mathrm{ml}$ digested plasmid DNA (lanes 2-9). Either $\mathrm{TauCl}$ or $\mathrm{TauBr}$ alone did not affect the structure of plasmid DNA (data not shown). TauCl (lanes 8, 9) did not affect the activity of DNAse. Interestingly, $\mathrm{TauBr}$ in contrast to $\mathrm{TauCl}$ inhibited the activity of DNAse. The inactivation of DNAse was observed when TauBr was added in excess to DNAse ( $\mathrm{TauBr}>500 \mu \mathrm{M})$.

\section{Discussion}

Oral biofilm (dental plaque) is a polymicrobial biofilm growing on hard and soft dental surfaces and is considered the main etiological factor for gingivitis and periodontitis [17]. Among a variety of oral bacteria, Streptococcus mutans and Porphyromonas gingivalis play a crucial role in the formation of dental plaque and in the pathogenesis of periodontal diseases, respectively [17, 18, 20-22]. Modern concepts for the prevention and therapy of biofilm-associated infections are based on eradication of the biofilm by using antibiotics combined 
A

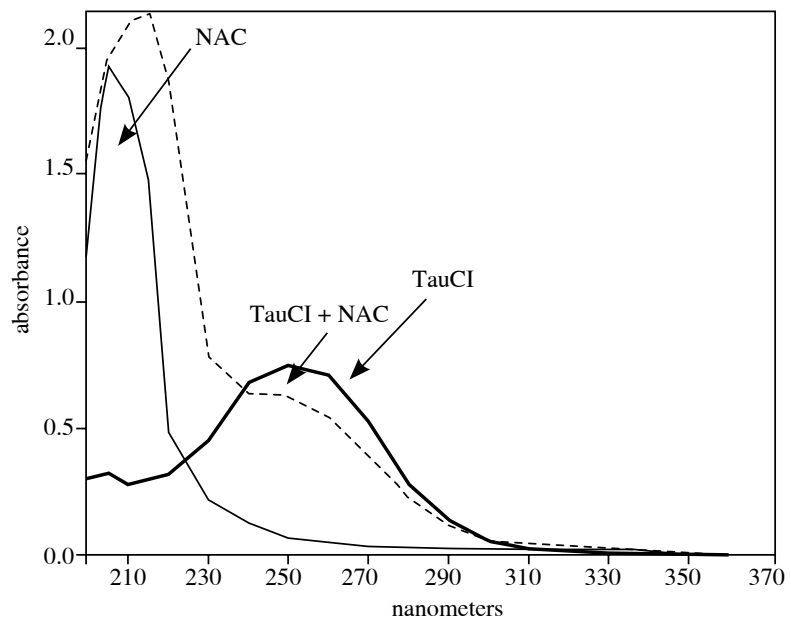

B

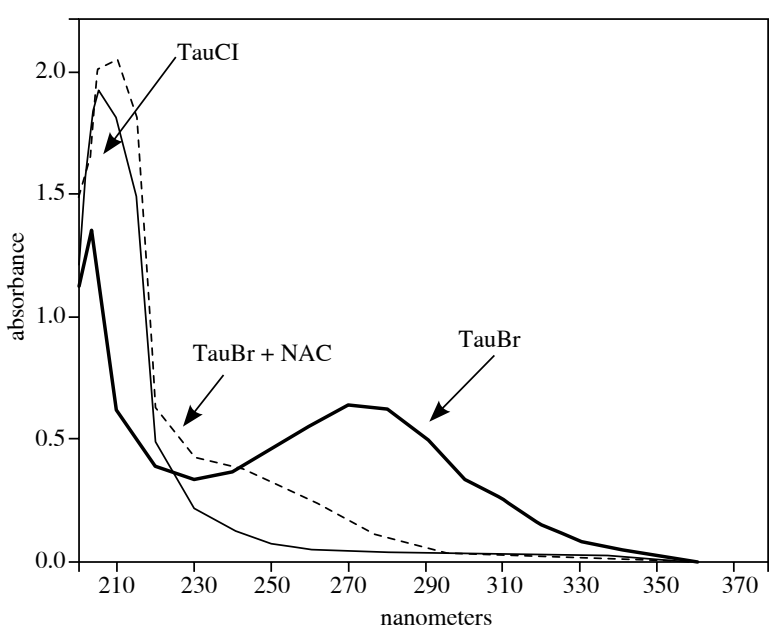

Fig. 6. Effect of $\mathrm{NAC}$ on the stability of $\mathrm{TauCl}(\mathrm{A})$ and $\mathrm{TauBr}(\mathrm{B})$ performed at $\mathrm{pH} 7,4$. TauCl, $\mathrm{TauBr}$ and $\mathrm{NAC}$ solutions were mixed in equimolar concentrations $(3 \mathrm{mM})$. The UV spectra were measured as described in methods

A

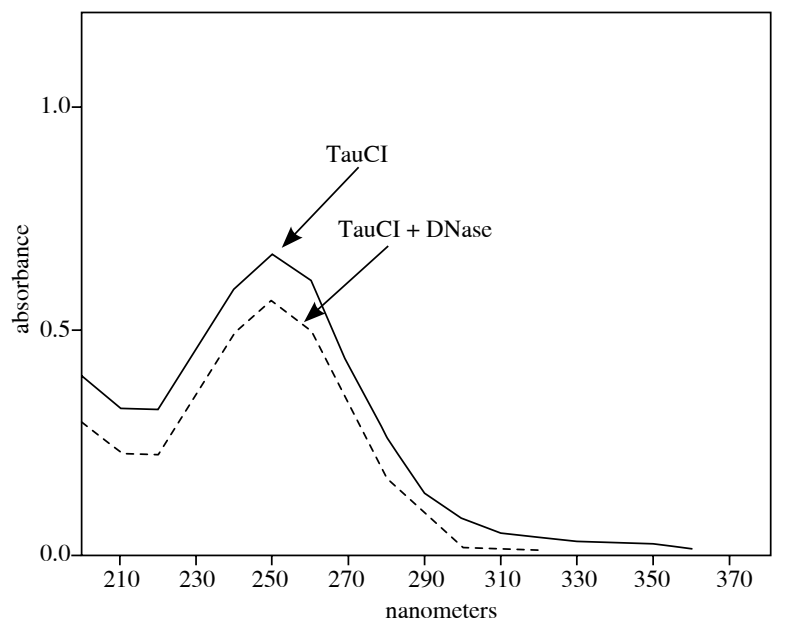

B

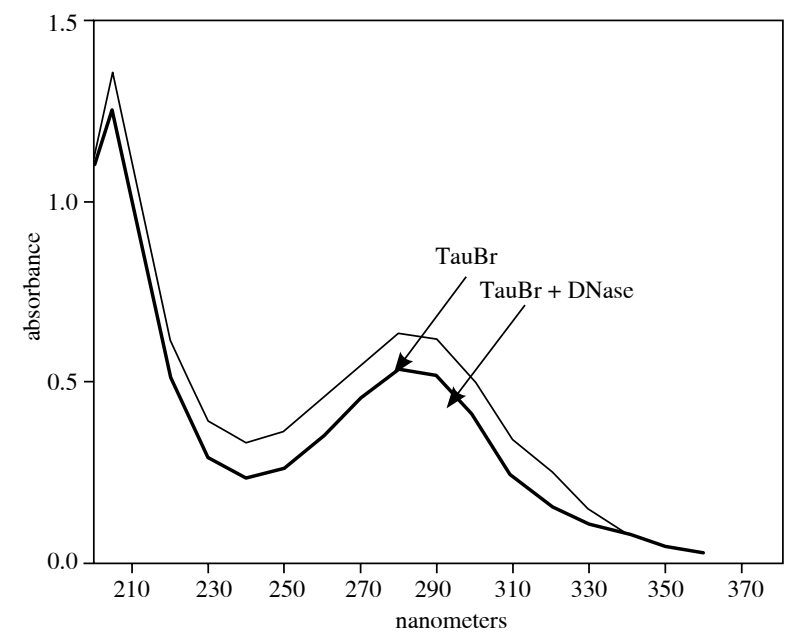

Fig. 7. Effect of DNase on the stability of $\mathrm{TauCl}(\mathrm{A})$ and $\mathrm{TauBr}(\mathrm{B})$ at $\mathrm{pH}$ 7.4. $\mathrm{TauCl}$, TauBr and DNase solutions were mixed in equimolar concentrations $(3 \mathrm{mM})$. The UV spectra were measured as described in methods

with adjuvant substances, capable of destroying biofilm matrix. Nevertheless, none of the currently available therapies are effective due to extreme resistance of biofilm bacteria to antimicrobial agents. It has been documented that bacteria within a biofilm can be even 1000 times more resistant to antibiotics than their planktonic counterparts [18]. To overcome this problem a variety of substances with a capacity to reduce biofilm growth has been tested, including NAC and DNase.

$\mathrm{N}$-acetylcysteine is a thiol (sulfhydryl groups) - containing antioxidant with antibacterial properties and is a precursor in the formation of the antioxidant glutathione in the body. NAC, due to its mucus-dissolving prop- erties, is used in medical treatment of chronic bronchitis. Recently, it has been found that NAC can increase therapeutic efficacy of antibiotics by degrading the extracellular polysaccharide matrix of biofilms [23-26]. However, the efficacy of NAC in reduction of dental plaque (oral biofilm bacteria) has not been tested yet. DNase, the second antibiofilm agent we have used in this study, also has the capacity to destroy components of the biofilm matrix, such as extracellular DNA. For example, the capacity of DNase to disrupt $P$. aeruginosa biofilm has been shown by Mohammed et al. [27, 28].

The main task of this study was to determine whether NAC or DNase can be used in combination with taurine 


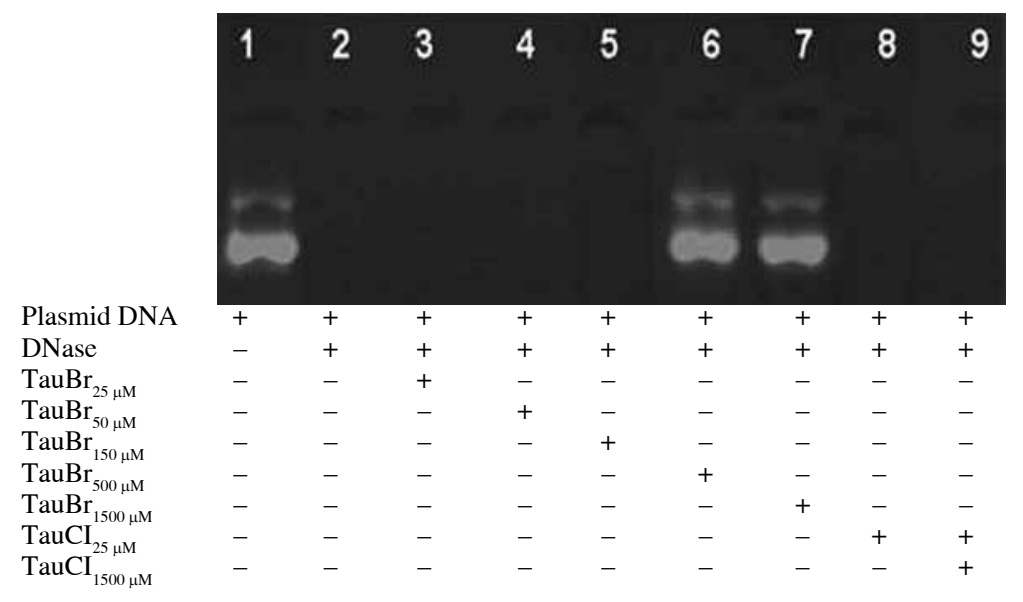

Fig. 8. Gel electrophoresis of plasmid DNA in the presence of DNAse at concentration of $50 \mathrm{mg} / \mathrm{ml}($ lanes 2-9) and TauCl at concentrations $25,1500 \mu \mathrm{M}$ (lanes 8, 9) or TauBr at concentrations 25, 50, 150, 500, $1500 \mu \mathrm{M}$ (lanes 3-7). Time of digestion of plasmid DNA was 40 minutes. Line 1 plasmid DNA without digestion

haloamines in the local treatment of biofilm-associated infections, especially in the treatment of periodontal diseases. We have chosen taurine haloamines ( $\mathrm{TauCl}$ and $\mathrm{TauBr}$ ) as previously our group and the others have shown their anti-inflammatory and antibacterial properties and promising potential in the local treatment of chronic (biofilm-associated) infectious diseases [13-16].

This study shows that at a neutral $\mathrm{pH}$ both monohaloamines, $\mathrm{TauCl}$ and $\mathrm{TauBr}$, can effectively kill the planktonic form of dental plaque bacteria, namely, S. mutans and $P$. gingivalis. However, $\mathrm{TauCl}$ antimicrobial activity was observed at very high concentrations. It indicates that $\mathrm{TauCl}$ is a relatively weak antiseptic agent. On the other hand, in acidic milieu ( $\mathrm{pH} 4-6)$, which is typical of an inflammatory environment, the ability of $\mathrm{TauCl}$ to kill pathogens increases significantly due to formation of a more potent $\mathrm{TauCl}_{2}$ (taurine dichloramine). $\mathrm{TauCl}_{2}$ has stronger bactericidal properties than $\mathrm{TauCl}$, especially against Gram-negative bacteria probably due to a better penetration into bacteria. In addition, transfer of the active chlorine (transchlorination) from $\mathrm{TauCl}$ to amino groups of other molecules enhances its activity, mainly because of the formation of monochloramine $\left(\mathrm{NH}_{2} \mathrm{Cl}\right)[29,30]$.

$\mathrm{TauBr}$, in contrast to $\mathrm{TauCl}$, shows the microbicidal activity at very low concentrations $<10 \mu \mathrm{M}$, even as taurine monobromamine. Therefore, these results, together with our previous study $[2,20]$, suggest that $\mathrm{TauBr}$ is a more promising candidate than $\mathrm{TauCl}$ for killing bacteria hidden in the biofilm matrix, but, as $\mathrm{TauCl}$ is a more stable molecule than $\mathrm{TauBr}$, we were tempted to examine the effect of anti-biofilm agents on activity of both taurine haloamines.

Previously, we have shown that $\mathrm{TauBr}$ but not $\mathrm{TauCl}$ is decomposed by $\mathrm{H}_{2} \mathrm{O}_{2}$ [7]. In this study, the low stabili- ty (high reactivity) of TauBr has been confirmed. Analysis of UV spectra clearly indicates that NAC completely decomposed TauBr without any effect on the stability of TauCl. The effect of NAC on TauBr may be explained by its antioxidant/scavenging properties. NAC is a powerful scavenger of some oxidants, such as hypochlorous acid and hydrogen peroxide [31]. In contrast to NAC, DNase, the second tested antibiofilm agent, did not affect the stability of either $\mathrm{TauCl}$ or TauBr. On the other hand, $\mathrm{TauBr}$ inhibited DNase enzymatic activity, when used at a concentration similar to that of DNase or in excess.

To prove whether NAC and DNAse are able to enhance anti-inflammatory properties of taurine haloamines, we also examined their ability to reduce ROS generation by activated inflammatory cells. Our previous [6, 7] and present results clearly indicate that both taurine haloamines can reduce generation of ROS by neutrophils (major cells of PECs) stimulated with opsonized zymosan (activation through FcR). The similar antioxidant effect of $\mathrm{TauCl}$ and TauBr was observed after stimulation of PECs with $P$. gingivalis and $S$. mutans (activation through TLRs) (Table 2). Therefore, we suggest that $\mathrm{TauCl}$ and $\mathrm{TauBr}$ may in vivo ameliorate inflammatory response induced by oral biofilm bacteria. Importantly, DNase did not show any effect on ROS production, while NAC confirmed its own antioxidant potential. However, the additive effect of NAC on antioxidant properties of $\mathrm{TauCl}$ in vitro was negligible, in our experimental set-up.

In conclusion, this preliminary study confirms previous suggestions that taurine haloamines, due to their anti-microbial and anti-inflammatory properties, are good candidates for a local treatment of periodontal diseases. It has become clear that they should be used in a combination with anti-biofilm agents to be more effective in 
killing of biofilm-associated bacteria (e.g. P. gingivalis in the subgingival biofilm). However, expected positive therapeutic efficacy of such combination treatment requires good stability of both components and synergistic or at least additive antimicrobial effect. Therefore, NAC can be combined with $\mathrm{TauCl}$ but not with $\mathrm{TauBr}$. On the other hand, the outstanding anti-microbial properties of $\mathrm{TauBr}$ indicate that $\mathrm{TauBr}$ with another adjuvant agent should be used in prevention and eradication of bacterial biofilms. Therefore, further studies should examine influence of matrix-degrading compounds on anti-inflammatory and anti-bacterial properties of taurine haloamines, especially on $\mathrm{TauBr}$, the more promising local antiseptic.

This paper was supported by a grant from the Jagiellonian University Medical College (K/ZDS/002964). The authors declare no conflict of interest.

\section{References}

1. Gottardi W, Nagl M (2002): Chemical properties of N-chlorotaurine sodium, a key compound in the human defence system. Arch Pharm 335: 411-421.

2. Marcinkiewicz J, Biedroń R, Białecka A, et al. (2006): Susceptibility of Propionibacterium acnes and Staphylococcus epidermidis to killing by MPO-halide system products. Implication for taurine bromamine as a new candidate for topical therapy in treating acne vulgaris. Arch Immunol Ther Exp 54: 61-68.

3. Marcinkiewicz J, Grabowska A, Bereta J, et al. (1998): Taurine chloramine down-regulates the generation of murine neutrophil inflammatory mediators. Immunopharmacology 40: 27-38.

4. Marcinkiewicz J, Grabowska A, Bereta J, et al. (1995): Taurine chloramine, a product of activated neutrophils, inhibits in vitro the generation of nitric oxide and other macrophage inflammatory mediators. J Leukoc Biol 1995; 58: 667-674.

5. Koprowski M, Marcinkiewicz J (2002): Taurine chloramine - its role in immunity and new perspectives for clinical use. Centr Eur J Immunol 27: 69-74.

6. Marcinkiewicz J, Kurnyta M, Biedroń R, et al. (2006): Anti-inflammatory effects of taurine derivatives (taurine chloramine, taurine bromamine, and taurolidine) are mediated by different mechanisms. Adv Exp Med Biol 583: 471-492.

7. Marcinkiewicz J, Mak M, Bobek M, et al. (2005): Is there a role of taurine bromamine in inflammation? Interactive effects with nitrite and hydrogen peroxide. Inflamm Res 54: 42-49.

8. Marcinkiewicz J, Walczewska M, Olszanecki R, et al. (2009): Taurine haloamines and heme oxygenase- 1 cooperate in the regulation of inflammation and attenuation of oxidative stress. Adv Exp Med Biol 643: 439-449.

9. Olszanecki R, Kurnyta M, Biedroń R, et al. (2008): The role of heme oxygenase- 1 in down regulation of PGE2 production by taurine chloramine and taurine bromamine in J774.2 macrophages. Amino Acids 35: 359-364.

10. Kim C, Cha YN (2009): Production of reactive oxygen and nitrogen species in phagocytes is regulated by taurine chloramine. Adv Exp Med Biol 643: 463-472.
11. Chorazy-Massalska M, Kontny E, Kornatka A, et al. (2004): The effect of taurine chloramine on pro-inflammatory cytokine production by peripheral blood mononuclear cells isolated from rheumatoid arthritis and osteoarthritis patients. Clin Exp Rheumatol 22: 692-698.

12. Kim C, Jang JS, Cho MR, et al. (2010): Taurine chloramine induces heme oxygenase-1 expression via Nrf2 activation in murine macrophages. Int Immunopharmacol 10: 440-446.

13. Neher A, Nagl M, Appenroth E, et al. (2004): Acute otitis externa: efficacy and tolerability of $\mathrm{N}$-chlorotaurine, a novel endogenous antiseptic agent. Laryngoscope 114: 850-854.

14. Gstöttner M, Nagl M, Pototschnig C, et al. (2003): Refractory rhinosinusitis complicating immunosuppression: application of $\mathrm{N}$-chlorotaurine, a novel endogenous antiseptic agent. ORL J Otorhinolaryngol Relat Spec 65: 303-305.

15. Marcinkiewicz J, Wojas-Pelc A, Walczewska M, et al. (2008): Topical taurine bromamine, a new candidate in the treatment of moderate inflammatory acne vulgaris: a pilot study. Eur J Dermatol 18: 433-439.

16. Mainnemare A, Mégarbane B, Soueidan A, et al. (2004): Acid and taurine-N-monochloramine in periodontal diseases. J Dent Res 3: 823-831.

17. Hřiby N, Ciofu O, Johansen HK, et al. (2011): Clinical impact of bacterial biofilms. Int J Oral Sci 3: 55-65.

18. Huang R, Li M, Gregory RL(2011): Bacterial interactions in dental biofilm. Virulence 2: 435-444.

19. Gacoń A. Influence of biofilm formation inhibitory factors on the microbial activity of taurine bromamine and taurine chloramine. Master Work 2011.

20. Marcinkiewicz J, Strus M, Walczewska M, et al. (2013): Influence of taurine haloamines ( $\mathrm{TauCl}$ and $\mathrm{TauBr}$ ) on the development of Pseudomonas aeruginosa biofilm: a preliminary study. Adv Exp Med Biol 775: 269-283.

21.Hojo K, Nagaoka S, Ohshima T, et al. (2009): Bacterial interactions in dental biofilm development. J Dent Res 88: 982990.

22.Cugini C, Klepac-Ceraj V, Rackaityte E, et al. (2013): Porphyromonas gingivalis: keeping the pathos out of the biont. J Oral Microbiol 5.

23. Aslam S, Darouiche RO (2011): Role of antibiofilm-antimicrobial agents in controlling device-related infections. Int J Artif Organs 34: 752-758.

24. Olofsson AC, Hermansson M, Elwing H (2003): N-acetyl-L-cysteine affects growth, extracellular polysaccharide production, and bacterial biofilm formation on solid surfaces. Appl Environ Microbiol 69: 4814-4822.

25.El-Feky MA, El-Rehewy MS, Hassan MA, et al. (2009): Effect of ciprofloxacin and $\mathrm{N}$-acetylcysteine on bacterial adherence and biofilm formation on ureteral stent surfaces. Pol J Microbiol 58: 261-267.

26. Quah SY, Wu S, Lui JN, et al. (2012): N-acetylcysteine inhibits growth and eradicates biofilm of Enterococcus faecalis. J Endod J 38: 81-5.

27. Ali Mohammed MM, Nerland AH, Al-Haroni M, et al. (2013): Characterization of extracellular polymeric matrix, and treatment of Fusobacterium nucleatum and Porphyromonas gingivalis biofilms with DNase I and proteinase K. J Oral Microbiol 5.

28.Parks QM, Young RL, Poch KR, et al. (2009): Neutrophil enhancement of Pseudomonas aeruginosa biofilm development: human F-actin and DNA as targets for therapy. J Med Microbiol 58 (Pt 4): 492-502. 
29. Gottardi W, Nagl M (2010): N-chlorotaurine, a natural antiseptic with outstanding tolerability. J Antimicrob Chemother 65: 399-409.

30. Gottardi W, Hagleitner M, Nagl M (2005): N,N-dichlorotaurine: chemical and bactericidal properties. Arch Pharm (Weinheim) 338: 473-483.

31. Aruoma OI, Halliwell B, Hoey BM, et al. (1989): The antioxidant action of $\mathrm{N}$-acetylcysteine: its reaction with hydrogen peroxide, hydroxyl radical, superoxide, and hypochlorous acid. Free Radic Biol Med 6: 593-597. 